\title{
On fractals which are not so terrible
}

\author{
by
}

António M. Caetano (Aveiro)

\begin{abstract}
The notion of NST domain and the closely related notion of ball condition, both topological in nature and quite useful within the theory of function spaces, are compared with each other (and with the older concept of porosity) and also with other notions of interest, like those of $d$-set and of interior regular domain, which have a measuretheoretical nature. Also, after extending the idea of NST (not so terrible) to a larger class of sets, the property is studied in the context of anisotropic self-affine fractals.
\end{abstract}

1. Introduction. In [3, pp. 143-144] Frazier and Jawerth introduced the concept of NST (not so terrible) domain in order to characterize a property of certain functions belonging to Triebel-Lizorkin spaces. This follows a long trend, not to be discussed here, of imposing conditions on (or near the boundary of) domains in order to guarantee certain properties for function spaces on those domains. The interested reader may want to consult [7], [8] and [5], as well as the references given there, for the older material.

One of our points here is that the main idea underlying the concept of NST domain has been repeatedly introduced after the work [3] (and even before it, under the name of porosity - cf. Section 3 below), maybe in disguised form. So, for example, under mild hypotheses on a (fractal-to-be) set $\Gamma$, saying that $\Gamma$ satisfies the so-called ball condition considered in $[9, \mathrm{p}$. 142 ] is equivalent to saying that $\Gamma^{\mathrm{c}}$ is a NST domain. We shall detail this below, which will eventually lead us to define what should be meant by a not so terrible (NST) fractal.

Afterwards we give a direct proof that $d$-sets in $\mathbb{R}^{n}$ (see [5, pp. 28-32] or $[9$, p. 5]) are not so terrible fractals (or, what is the same, satisfy the ball condition) when $d<n$.

Next we study a subclass of the family of self-affine fractals and give sufficient conditions for an element of the subclass to be NST. Finally, we

2000 Mathematics Subject Classification: 28A80, 46E35.

Research partially supported by Instituto de Cooperação Científica e Tecnológica Internacional (Protocolo ICCTI/DAAD). 
exhibit an example of a self-affine fractal which is really terrible (that is, which is not NST), though it might not look like that at first glance.

\section{Preliminary discussion}

Definition 2.1 ([3, pp. 143-144]). A domain (i.e., a non-empty open set) $\Omega$ of $\mathbb{R}^{n}$ is said to be $N S T$ (not so terrible), or to be in NST (thinking about NST as a denomination for the class of all NST domains in $\mathbb{R}^{n}$, for some fixed $n \in \mathbb{N}$ ) if there exists $\mu \in \mathbb{N}$ with the property that for any dyadic cube $Q$ with side-length $\ell(Q) \leq 1$ and $\bar{Q} \cap \partial \Omega \neq \emptyset$, there exists a dyadic cube $P \subset Q$ with $\ell(P)=2^{-\mu} \ell(Q)$ such that $\stackrel{\circ}{P} \cap \partial \Omega=\emptyset$.

Of course, we will rule out, because of lack of interest, the case when $\Omega=\mathbb{R}^{n}$, that is, we shall only consider proper domains.

In the above definition, $\stackrel{\circ}{P}, \bar{Q}$ and $\partial \Omega$ stand, respectively, for the interior of $P$, the closure of $Q$ and the boundary of $\Omega$, as usual. A dyadic cube $Q$ in $\mathbb{R}^{n}$ is a cube

$$
Q_{\nu k} \equiv\left\{\left(x_{1}, \ldots, x_{n}\right): 2^{-\nu} k_{i} \leq x_{i}<2^{-\nu}\left(k_{i}+1\right) \text { for } i=1, \ldots, n\right\}
$$

for some $\nu \in \mathbb{Z}$ and $k \in \mathbb{Z}^{n}$. In particular, for each such $\nu$, the family $\left\{Q_{\nu k}: k \in \mathbb{Z}^{n}\right\}$ constitutes a tessellation (without intersections) of $\mathbb{R}^{n}$.

In this paper, the word cube, even if not dyadic, is always used in the sense of cube with sides parallel to the axes.

The simplest examples of proper NST domains are the domains $\Omega$ where $\partial \Omega$ has just a finite set of points. On the other hand, if $\partial \Omega$ is "too crowded" then surely $\Omega$ is not NST. However, NST domains do not have a clear relationship with the cardinality of their boundaries: for example, as will become clear along this paper, if $\partial \Omega$ is the Cantor set, then $\Omega$ is NST, though the Cantor set is uncountable; nevertheless, there are domains $\Omega$ not in NST for which $\partial \Omega$ is countable. We give an example in $\mathbb{R}$ :

Let $\partial \Omega \equiv\{0\} \cup\{1 / m: m \in \mathbb{N}\}$. Then $\Omega \notin$ NST. In fact, assume that such an $\Omega$ were in NST. Consider the sequence of dyadic cubes $Q_{\nu 1}, \nu \in \mathbb{N}$, which clearly intersect $\partial \Omega$, and a corresponding sequence of dyadic cubes $P(\nu) \subset Q_{\nu 1}$ with $\ell(P(\nu))=2^{-\mu} \ell\left(Q_{\nu 1}\right)$ and $P(\nu) \cap \partial \Omega=\emptyset$. It is clear from the structure of $\partial \Omega$ that $\ell(P(\nu))$ must not exceed $1 / 2^{\nu-1}-1 /\left(2^{\nu-1}+1\right)$, that is, $2^{-\nu+1} /\left(2^{\nu-1}+1\right)$, while $\ell\left(Q_{\nu 1}\right)=2^{-\nu}$. Therefore $0<2^{-\mu}=$ $\ell(P(\nu)) / \ell\left(Q_{\nu 1}\right) \leq 2 /\left(2^{\nu-1}+1\right) \rightarrow 0$ as $\nu$ goes to infinity, which is absurd!

\section{NST and the ball condition}

Definition 3.1. A non-empty subset $\Gamma$ of $\mathbb{R}^{n}$ such that $\stackrel{\circ}{\Gamma}=\emptyset$ is said to satisfy the ball condition if

$$
\begin{aligned}
\exists \eta \in] 0,1[: \forall x \in \Gamma, \forall r \in] & 0,1[, \exists y \\
B(y, \eta r) & \subset B(x, r) \text { and } B(y, \eta r) \cap \bar{\Gamma}=\emptyset,
\end{aligned}
$$


where $B(z, s)$ stands for the closed (Euclidean) ball centred at $z$ with radius $s$.

This is a slight modification of the definition presented in [9, p. 142], where $\Gamma$ was assumed to be Borel with zero Lebesgue measure. We decided to strip the definition off of any allusion to measure theory (and, since $|\Gamma|=0 \Rightarrow \stackrel{\circ}{\Gamma}=\emptyset$, we are retaining all the sets considered to satisfy the ball condition according the definition given in [9]). Observe that the assumption $\stackrel{\circ}{\Gamma}=\emptyset$ is quite natural, because if $\stackrel{\circ}{\Gamma} \neq \emptyset$ then the statement with quantifiers in the definition cannot be fulfilled. Actually, it can even be proved that this statement also implies that $|\bar{\Gamma}|=0$. This is an immediate consequence of the fact that such a $\bar{\Gamma}$ has Hausdorff dimension strictly less than $n$, which in turn follows from the fact that $\bar{\Gamma}$ has strong porosity bounded away from 0 at all of its points, in the sense explained in $[6$, p. 156].

We also mention that a set satisfying the ball condition is a particular kind of strongly porous set (cf. [6, p. 156]) and that, according to [6, p. 158], the notion of porosity goes back, at least in its weakest forms, to a 1920 work of Denjoy.

The following result can be proved by straightforward arguments:

Proposition 3.2. Let $\Gamma$ be a non-empty closed subset of $\mathbb{R}^{n}$ with $\stackrel{\circ}{\Gamma}=\emptyset$. Then $\Gamma$ satisfies the ball condition if, and only if, $\Gamma^{\mathrm{c}} \in \mathrm{NST}$.

REMARK 3.3. Actually, the above statement is valid even without the assumption that $\Gamma$ is closed, as long as we replace " $\Gamma^{\mathrm{c}} \in \mathrm{NST}$ " by

"there exists $\mu \in \mathbb{N}$ with the property that for any dyadic cube $Q$ with side-length $\ell(Q) \leq 1$ and $\bar{Q} \cap \bar{\Gamma} \neq \emptyset$, there exists a dyadic cube $P \subset Q$ with $\ell(P)=2^{-\mu} \ell(Q)$ such that $\stackrel{\circ}{P} \cap \bar{\Gamma}=\emptyset "$,

where we can, alternatively, write $\partial\left(\Gamma^{\mathrm{c}}\right)$ or $\partial \Gamma$ for $\bar{\Gamma}$.

The similarity between the quotation written above (with the possibility of replacing $\bar{\Gamma}$ by $\partial \Gamma$ ) and the definition of a NST domain prompts the following extension of Definition 2.1:

DeFinition 3.4. Let $\Gamma$ be a non-empty subset of $\mathbb{R}^{n}$ such that either $\stackrel{\circ}{\Gamma}=\Gamma$ or $\stackrel{\circ}{\Gamma}=\emptyset$. The set $\Gamma$ is said to be $N S T$ (not so terrible), or to be in NST, if there exists $\mu \in \mathbb{N}$ with the property that for any dyadic cube $Q$ with side-length $\ell(Q) \leq 1$ and $\bar{Q} \cap \partial \Gamma \neq \emptyset$, there exists a dyadic cube $P \subset Q$ with $\ell(P)=2^{-\mu} \ell(Q)$ such that $\stackrel{\circ}{P} \cap \partial \Gamma=\emptyset$.

REMARK 3.5. (i) Of course, in the case of $\stackrel{\circ}{\Gamma}=\emptyset$ we already know that $\partial \Gamma$ in the above definition can be replaced by $\partial\left(\Gamma^{\mathrm{c}}\right)$ or $\bar{\Gamma}$. Also in that case, the remark above shows that $\Gamma \in \mathrm{NST}$ if, and only if, $\Gamma$ satisfies the ball condition. 
(ii) With the above definition it is easy to see that a proper domain belongs to NST if, and only if, its boundary does; or, equivalently, if, and only if, its boundary satisfies the ball condition.

We do not want to go into details, but we exhibit the relationship between the concept of NST set just introduced and the concept of open set condition as given in [10] (see there for the definition): it is not difficult to see that a non-empty subset $\Gamma$ of $\mathbb{R}^{n}$ with $\stackrel{\circ}{\Gamma}=\emptyset$ belongs to NST if, and only if, it satisfies that open set condition (except possibly the assumption that $\Gamma$ is Borel, made in [10]). Be aware, however, that the notion of open set condition used in [10] is different from the notion with the same name which we are going to consider later in this paper.

4. NST and $d$-sets. We recall the notion of $d$-set [5, pp. 28-32]:

Definition 4.1. Let $\Gamma$ be a non-empty closed subset of $\mathbb{R}^{n}$ and $d \in$ ]0, $n] . \Gamma$ is said to be a $d$-set if

$$
\exists c_{1}, c_{2}>0: \forall \gamma \in \Gamma, \forall 0<r \leq 1, \quad c_{1} r^{d} \leq \mathcal{H}^{d}(B(\gamma, r) \cap \Gamma) \leq c_{2} r^{d},
$$

where $\mathcal{H}^{d}$ stands for the $d$-dimensional Hausdorff measure on $\mathbb{R}^{n}$.

REMARK 4.2. (i) A $d$-set has Hausdorff dimension equal to $d$.

(ii) It does not matter whether we use the Euclidean balls above or balls for the infinity norm (i.e., cubes), or substitute $0<r \leq 1$ by $0<r \leq r_{0}$, for some fixed $r_{0}>0$, in the sense that these modifications do not change the class of sets to which the definition applies.

This concept of $d$-set has been found very convenient when dealing with function spaces on closed sets (see, for example, [5] and [9]). Be aware that the expression " $d$-set" has also been used with a different meaning in Fractal Geometry (cf., for example, [1]).

The following result can be derived from an assertion presented (without proof) in [4, Prop. 2]. We give a direct proof below.

Proposition 4.3. Let $\Gamma$ be a d-set with $d<n$. Then $\Gamma \in$ NST.

Proof. From the properties of Hausdorff measure, it is clear that $\stackrel{\circ}{\Gamma}=\emptyset$.

Let $Q_{\nu}$ be a dyadic cube with side-length $2^{-\nu} \leq 1$ (that is, with $\nu \in \mathbb{N}_{0}$ ) and with $\bar{Q}_{\nu} \cap \Gamma \neq \emptyset$. For each $\varrho \in \mathbb{N}$ consider the tessellation of $Q_{\nu}$ by dyadic subcubes $q$ with side-length $2^{-(\nu+\varrho)}$. Clearly, there are $2^{\varrho n}$ such subcubes.

Pick up only the subcubes $q$ of that tessellation such that $\bar{q} \cap \Gamma \neq \emptyset$ and put them in some order: $q_{1}, \ldots, q_{n_{\varrho}}$. In particular, $n_{\varrho}$ stands for the number of such smaller cubes. Next form a subsequence $\left(q_{\sigma(r)}\right)_{r}$ using the following criteria (where $c q$ stands for the cube with the same centre of $q$ but with $c$ times its side-length):

(i) $q_{1}$ is the first element of the subsequence; 
(ii) if $q_{\sigma(r)}$ is an element of the subsequence and

$$
J_{r} \equiv\left\{j \in \mathbb{N}: \forall s=1, \ldots, r, 3 \stackrel{\circ}{q(s)}_{\sigma} \cap q_{\sigma(r)+j}=\emptyset\right\} \neq \emptyset,
$$

then $q_{\sigma(r+1)} \equiv q_{\sigma(r)+\min J_{r}}$;

(iii) the subsequence terminates when $J_{r}=\emptyset$.

Denote by $m_{\varrho}$ the number of cubes in this subsequence. It is clear that, for each cube $q$ of the original sequence which remains in the subsequence, we may be throwing away as many cubes as the number of cubes of the tessellation which are neighbours of $q$ (but no more than that). Since the number of such neighbours is at most $3^{n}-1$, we can write

$$
n_{\varrho} \leq m_{\varrho}+m_{\varrho}\left(3^{n}-1\right)=3^{n} m_{\varrho} .
$$

Recall that each $\bar{q}_{\sigma(r)}$ contains an element of $\Gamma$. Fix one such element (call it $x_{r}$ ) in each $\bar{q}_{\sigma(r)}$ and denote by $K_{r}$ the closed cube centred at $x_{r}$ and with side-length $2^{-(\nu+\varrho+1)}$. Observe that $K_{r} \subset 2 \dot{\circ}_{\sigma(r)}$ and the $m_{\varrho}$ cubes $2 \stackrel{q}{\sigma(r)}$ are pairwise disjoint.

Fix also a point in $\bar{Q}_{\nu} \cap \Gamma$ and consider the closed cube $K$ centred at that point and with side-length $3 \times 2^{-\nu}$, so that, in particular, $\bigcup_{r=1}^{m_{\varrho}} 2 \stackrel{\circ}{q}_{\sigma(r)} \subset K$.

Now we use the hypothesis that $\Gamma$ is a $d$-set in order to write

$$
\begin{aligned}
c_{2}\left(3 \times 2^{-\nu-1}\right)^{d} & \geq \mathcal{H}^{d}(K \cap \Gamma) \\
& \geq \mathcal{H}^{d}\left(\bigcup_{r=1}^{m_{\varrho}} 2 \stackrel{\circ}{\sigma}_{\sigma(r)} \cap \Gamma\right)=\sum_{r=1}^{m_{\varrho}} \mathcal{H}^{d}(2 \stackrel{\circ}{\sigma(r)} \cap \Gamma) \\
& \geq \sum_{r=1}^{m_{\varrho}} \mathcal{H}^{d}\left(K_{r} \cap \Gamma\right) \geq \sum_{r=1}^{m_{\varrho}} c_{1} 2^{-(\nu+\varrho+2) d} \\
& =m_{\varrho} c_{1} 2^{-\nu d_{2}} 2^{-(\varrho+2) d},
\end{aligned}
$$

from which it follows, by (2), that

$$
n_{\varrho} \leq c_{3} 2^{\varrho d},
$$

where $c_{3}>0$ depends only on $n, d$ and $\Gamma$.

Since there are exactly $2^{\varrho n}$ dyadic cubes of side-length $2^{-(\nu+\varrho)}$ in $Q_{\nu}$, the number of such cubes $q$ with $\bar{q} \cap \Gamma=\emptyset$ is $2^{\varrho n}-n_{\varrho}$. Using (3) we can then guarantee that the number of such cubes is bounded below by $2^{\varrho n}-c_{3} 2^{\varrho d}$. As this goes to infinity with $\varrho$ (here we have used the hypothesis $d<n$ ), we can be sure that there is a value $\varrho_{0}$ of $\varrho$ (depending only on $n, d$ and $\Gamma$ ) such that there is at least one dyadic subcube $q$ of side-length $2^{-\varrho_{0}} \ell\left(Q_{\nu}\right)$ in $Q_{\nu}$ such that $\stackrel{q}{\mathrm{q}} \cap \Gamma=\emptyset$.

This completes the proof that $\Gamma \in \operatorname{NST}$ (with $\mu=\varrho_{0}$ in Definition 3.4). 
REMARK 4.4. Even if one considers only not so terrible sets $\Gamma$ which are closed, there are some which are not $d$-sets, for any $d$. Take, for example, $\Gamma=\{0\} \cup\left\{2^{-m}: m \in \mathbb{N}_{0}\right\}$ in $\mathbb{R}$. Of course, no open NST set can be a $d$-set with $d<n$ (even if we omit the assumption of being closed in the definition of $d$-set, as we will do whenever necessary). Observe also that there are open NST sets which are not open $n$-sets (consider, for example, a domain with an outward cusp, as in Fig. 5 of $[11$, p. 657]). On the positive side, it is not difficult to see that, given a $\operatorname{NST}$ domain $\Omega$, the domain $(\partial \Omega)^{\mathrm{c}}$ is an open $n$-set.

Clearly, an $n$-set might not be a NST set in the sense of Definition 3.4: a simple example is given by a closed cube in $\mathbb{R}^{3}$, just because it is neither an open set nor has empty interior. However, the difference between the two concepts is deeper: if one tries to apply (1) to open instead of closed sets, one might find open sets which are $n$-sets in this modified sense but which do not belong to NST: a simple example is given by the open $\Gamma$ in $\mathbb{R}$ such that $\Gamma^{\mathrm{c}}=\partial \Gamma=\{0\} \cup\{1 / m: m \in \mathbb{N}\}$ (recall that we have already proved that such a $\Gamma$ is not NST - see Section 2). We give a more complicated example in two dimensions later on. As for $n$-sets (closed or not) $\Gamma$ such that $\stackrel{\circ}{\Gamma}=\emptyset$, they cannot be in NST: on the one hand, being $n$-sets, they must satisfy $|\Gamma|>0$; on the other hand, if this is true then $\Gamma$ cannot satisfy the ball condition (see comments after Definition 3.1); since $\stackrel{\circ}{\Gamma}=\emptyset$, it follows then by Remark 3.5(i) that $\Gamma \notin \mathrm{NST}$.

We also mention that a domain $\Omega$ in $\mathbb{R}^{n}$ is an open $n$-set if, and only if, it is an interior regular domain in the sense of [11], except that we do not assume boundedness of $\Omega$ and the equality $\Omega=\bar{\Omega}$, required there. More explicitly, if, and only if, there exists a positive $c$ with the property that for any $x \in \partial \Omega$ and any cube $Q$ centred at $x$ with side-length at most 1 , $|\Omega \cap Q| \geq c|Q|$.

5. NST and self-affine fractals. Let $N \geq 2$ be a natural number and let $A_{1}, \ldots, A_{N}$ be affine contractions on $\mathbb{R}^{n}$. That is, for each $l=1, \ldots, N$, $A_{l}$ is an affine map, i.e.,

$$
A_{l} x=T_{l} x+b_{l}, \quad \forall x \in \mathbb{R}^{n},
$$

where $b_{l} \in \mathbb{R}^{n}$ and $T_{l}$ is a linear transformation on $\mathbb{R}^{n}$, and $A_{l}$ is a contraction, i.e.,

$$
\exists 0<c_{l}<1: \quad\left|A_{l} x-A_{l} y\right| \leq c_{l}|x-y|, \quad \forall x, y \in \mathbb{R}^{n} .
$$

Of course, in view of (4), the last property is equivalent to the existence of $0<c_{l}<1$ such that $\left|T_{l} x\right| \leq c_{l}|x|$ for all $x \in \mathbb{R}^{n}$, or just to the statement that $\left\|T_{l}\right\|<1$. 
Definition 5.1. Given $N$ affine contractions $A_{1}, \ldots, A_{N}$, the unique non-empty compact subset $\Gamma$ of $\mathbb{R}^{n}$ satisfying the equality

$$
\Gamma=\bigcup_{l=1}^{N} A_{l} \Gamma
$$

is called the self-affine fractal generated by those $N$ maps.

REMARK 5.2. (i) The word "fractal" is being used loosely here-we could as well have written "set" instead.

(ii) This definition includes a proposition, namely that there is one and only one non-empty compact set $\Gamma$ satisfying (5). This is indeed the case: see $[2$, p. 114], where it is also shown that $\Gamma$ can be obtained as

$$
\Gamma=\bigcap_{k=1}^{\infty} A^{k} E
$$

for any non-empty compact subset $E$ of $\mathbb{R}^{n}$ such that $A_{l} E \subset E$ for $l=$ $1, \ldots, N$. In (6), $A^{k} E$ is defined in the following way:

$$
A^{0} E=E ; \quad A E=\bigcup_{l=1}^{N} A_{l} E ; \quad A^{k} E=A\left(A^{k-1} E\right), \quad \forall k \in \mathbb{N} .
$$

(iii) For examples and pictures, the interested reader is referred to [2].

The case when the affine contractions $A_{1}, \ldots, A_{N}$ are similarities, that is, satisfy

$$
\left|A_{l} x-A_{l} y\right|=c_{l}|x-y|, \quad \forall x, y \in \mathbb{R}^{n},
$$

for some $0<c_{l}<1, l=1, \ldots, N$, is well studied, mainly if those affine contractions are also supposed to satisfy the open set condition, which means that there exists a non-empty open set $\mathcal{U}$ in $\mathbb{R}^{n}$ such that

$$
A \mathcal{U} \subset \mathcal{U}, \quad A_{l} \mathcal{U} \cap A_{h} \mathcal{U}=\emptyset \quad \text { for } l \neq h
$$

(with $A$ as defined in (7)). Thus, the following is known (see [6, p. 67]):

Proposition 5.3. Let $A_{1}, \ldots, A_{N}$ be similarities satisfying the open set condition. Then the self-affine fractal generated by these contractions is a $d$-set, where $d$ is the unique positive number satisfying

$$
\sum_{l=1}^{N} c_{l}^{d}=1
$$

(cl as in (8)).

In the case when $d<n$ we can immediately conclude, on the basis of Proposition 4.3, that a self-affine fractal as in the preceding proposition is NST. 
Our next aim is to consider a class of self-affine fractals of sufficient interest (and where the contractions are not necessarily similarities) and establish easily verifiable conditions which guarantee that a particular element of that class is NST. Since we shall also show that not all elements of such a class are NST, what follows can also be considered as a contribution to understand how far the class of self-affine fractals is from the class NST.

Let $Q$ be the closed unit cube in $\mathbb{R}^{n}$ :

$$
Q \equiv\left\{x \equiv\left(x_{j}\right)_{j=1}^{n}: 0 \leq x_{j} \leq 1, \forall j=1, \ldots, n\right\} .
$$

Our class of self-affine fractals should contain only those which are generated by surjective ( $\Leftrightarrow$ injective in this context) affine contractions $A_{l}$, $l=1, \ldots, N$, such that

$$
\begin{gathered}
A_{l} Q \subset Q, \quad l=1, \ldots, N, \\
A_{l} \stackrel{\circ}{Q} \cap A_{m} \stackrel{\circ}{Q}=\emptyset \quad \text { if } l \neq m ; l, m=1, \ldots, N,
\end{gathered}
$$

and

$$
\sum_{l=1}^{N}\left|A_{l} Q\right|<1,
$$

where here $|\cdot|$ stands for Lebesgue measure in $\mathbb{R}^{n}$.

According to our Remark 5.2(ii) and (10), the self-affine fractal generated by a family of $A_{l}$ as above is $\Gamma$ given by

$$
\Gamma=\bigcap_{k=1}^{\infty} A^{k} Q,
$$

where $A^{k}$ has the same meaning as in (7). In particular,

$$
A^{k} Q=\bigcup_{1 \leq l_{1}, \ldots, l_{k} \leq N} A_{l_{1}} \ldots A_{l_{k}} Q, \quad k \in \mathbb{N} .
$$

Note that the hypotheses on $A_{l}$ also imply that $\Gamma$ satisfies the open set condition (9) with $\mathcal{U}=\stackrel{Q}{\text {. }}$

LEMma 5.4. Each self-affine fractal of the class introduced above has zero Lebesgue measure.

Proof. We have

$$
\begin{aligned}
0 \leq|\Gamma| & \leq\left|A^{k} Q\right| \leq \sum_{1 \leq l_{1}, \ldots, l_{k} \leq N}\left|A_{l_{1}} \ldots A_{l_{k}} Q\right| \\
& =\sum_{1 \leq l_{1}, \ldots, l_{k} \leq N}\left|\operatorname{det} T_{l_{1}}\right| \ldots\left|\operatorname{det} T_{l_{k}}\right| \\
& =\left(\sum_{1 \leq l_{1} \leq N}\left|\operatorname{det} T_{l_{1}}\right|\right) \ldots\left(\sum_{1 \leq l_{k} \leq N}\left|\operatorname{det} T_{l_{k}}\right|\right)=r^{k}
\end{aligned}
$$


where $r \equiv \sum_{l=1}^{N}\left|A_{l} Q\right|<1$ by (12). The conclusion follows immediately by letting $k$ go to $\infty$.

Remark 5.5. As a consequence, such sets $\Gamma$ have empty interior. Therefore, a set $\Gamma$ of that class is NST if, and only if, it satisfies the ball condition (cf. Remark 3.5(i)).

The above class is still too large for what we intend to do. We restrict further attention to the so-called anisotropic fractals, in the sense of $[9$, p. 14] (though here $n$ can be any natural number). For these, the affine contractions $A_{l}$ have the form

$$
A_{l}:\left(x_{j}\right)_{j=1}^{n} \mapsto\left(\eta_{j}^{(l)} r_{j}^{(l)} x_{j}\right)_{j=1}^{n}+\left(b_{j}^{(l)}\right)_{j=1}^{n},
$$

where $\left.\eta_{j}^{(l)} \in\{-1,1\}, r_{j}^{(l)} \in\right] 0,1\left[\right.$ and $b_{j}^{(l)}, j=1, \ldots, n$, are given constants for each $l=1, \ldots, N$.

THEOREM 5.6. Let $\Gamma$ be an anisotropic self-affine fractal. Let $A_{l}, l=$ $1, \ldots, N$, denote the affine contractions (13) which generate $\Gamma$. Assume that for every $i \in\{1, \ldots, n\}$, every $l \in\{1, \ldots, N\}$ and every $x \equiv\left(x_{j}\right)_{j=1}^{n} \in A_{l} Q$, there exist $y \equiv\left(x_{1}, \ldots, x_{i-1}, y_{i}, x_{i+1}, \ldots, x_{n}\right)$ and an open cube $q \subset Q$ such that $y \in \bar{q}$ and

$$
q \cap \bigcup_{\ell=1}^{N} A_{\ell} Q=\emptyset .
$$

Then $\Gamma$ is NST.

Proof. Step 1. Observe that there is a $\mu>0$ such that the side-length of any $q$ as above can be taken to be $\geq \mu$. Also, given that $q$ is open, $q \subset Q$

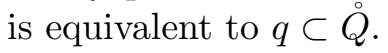

According to Remark 5.5, we must prove that $\Gamma$ satisfies the ball condition.

Consider any

$$
x \equiv\left(x_{j}\right)_{j=1}^{n} \in \Gamma=\bigcap_{k=1}^{\infty} A^{k} Q=\bigcap_{k=1}^{\infty} \bigcup_{1 \leq l_{1}, \ldots, l_{k} \leq N} A_{l_{1}} \ldots A_{l_{k}} Q
$$

and any closed ball $B(x, r)$ with $r \in] 0,1\left[\right.$. Note that $x \in A^{k} Q$ for all $k \in \mathbb{N}$, and for each $k$ considered, $x$ belongs to some $A_{l_{1}} \ldots A_{l_{k}} Q$, where $1 \leq l_{1}, \ldots, l_{k} \leq N$.

Step 2. Let

$$
\begin{aligned}
\alpha & \equiv \min \left\{r_{j}^{(l)}: j \in\{1, \ldots, n\}, l \in\{1, \ldots, N\}\right\}, \\
\beta & \equiv \max \left\{r_{j}^{(l)}: j \in\{1, \ldots, n\}, l \in\{1, \ldots, N\}\right\}, \\
\theta & \equiv(\alpha \beta) /(\sqrt{n} \alpha+\beta) .
\end{aligned}
$$


Note that $\theta<\alpha$, whence $r_{j}^{(l)}>\theta r$ for all $j \in\{1, \ldots, n\}$ and $l \in\{1, \ldots, N\}$. On the other hand, the sequence $\left(r_{j}^{\left(l_{1}\right)} \ldots r_{j}^{\left(l_{m}\right)}\right)_{m \in \mathbb{N}}$, viewed as a sequence of functions of $\left(l_{s}\right)_{s \in \mathbb{N}}$ and $j$, tends to zero uniformly on the set $\left\{\left(\left(l_{s}\right)_{s \in \mathbb{N}}, j\right)\right.$ : $\left.\left(l_{s}\right)_{s \in \mathbb{N}} \subset\{1, \ldots, N\}, j \in\{1, \ldots, n\}\right\}$, which follows from the inequalities $0<r_{j}^{\left(l_{1}\right)} \ldots r_{j}^{\left(l_{m}\right)} \leq \beta^{m}$ and $\beta<1$. Therefore it is possible to find $k \in \mathbb{N}$ such that $r_{j}^{\left(l_{1}\right)} \ldots r_{j}^{\left(l_{k}\right)} \leq \theta r$ for any such $j$ and $\left(l_{s}\right)_{s \in \mathbb{N}}$.

Fix now $l_{1}, \ldots, l_{k}$ in such a way that for the given $x$ we have

$$
x \in A_{l_{1}} \ldots A_{l_{k}} Q .
$$

Since, for each $j, r_{j}^{\left(l_{1}\right)}>\theta r$ and $r_{j}^{\left(l_{1}\right)} \ldots r_{j}^{\left(l_{k}\right)} \leq \theta r$, there is a first natural $k_{j} \in[2, k]$ such that the product $r_{j}^{\left(l_{1}\right)} \ldots r_{j}^{\left(l_{k_{j}}\right)}$ becomes $\leq \theta r$. Let $k_{0} \equiv$ $\min _{1 \leq j \leq n} k_{j}$, so that $r_{j}^{\left(l_{1}\right)} \ldots r_{j}^{\left(l_{k_{0}-1}\right)}>\theta r$ for all $j \in\{1, \ldots, n\}$. Among all $j$ 's such that $r_{j}^{\left(l_{1}\right)} \ldots r_{j}^{\left(l_{k_{0}}\right)} \leq \theta r$ choose one - call it $i$ - such that the product is minimum. For convenience, we shall denote $k_{0}$ again by $k$. So, in what follows the $k \in \mathbb{N}$ and $i \in\{1, \ldots, n\}$ are chosen such that

$$
r_{i}^{\left(l_{1}\right)} \ldots r_{i}^{\left(l_{k}\right)} \leq \theta r
$$

and

(16) $\forall j \in\{1, \ldots, n\}, \quad r_{j}^{\left(l_{1}\right)} \ldots r_{j}^{\left(l_{k-1}\right)}>\theta r, \quad r_{j}^{\left(l_{1}\right)} \ldots r_{j}^{\left(l_{k}\right)} \geq r_{i}^{\left(l_{1}\right)} \ldots r_{i}^{\left(l_{k}\right)}$.

Step 3. Note that (14) still holds for this new $k$ (this is an easy consequence of (10)), so that there exists $x^{\prime} \equiv\left(x_{j}^{\prime}\right)_{j=1}^{n} \in A_{l_{k}} Q$ such that $x=$ $A_{l_{1}} \ldots A_{l_{k-1}} x^{\prime}$. From the hypothesis, there exists $y^{\prime}=\left(x_{1}^{\prime}, \ldots, x_{i-1}^{\prime}, y_{i}^{\prime}, x_{i+1}^{\prime}\right.$,

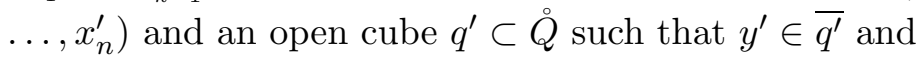

$$
q^{\prime} \cap \bigcup_{l=1}^{N} A_{l} Q=\emptyset
$$

from which it follows that

$$
A_{l_{1}} \ldots A_{l_{k-1}} q^{\prime} \subset A_{l_{1}} \ldots A_{l_{k-1}} \stackrel{\circ}{ }
$$

and

$$
\left(A_{l_{1}} \ldots A_{l_{k-1}} q^{\prime}\right) \cap \bigcup_{l=1}^{N} A_{l_{1}} \ldots A_{l_{k-1}} A_{l} Q=\emptyset .
$$

As a consequence,

$$
\left(A_{l_{1}} \ldots A_{l_{k-1}} q^{\prime}\right) \cap \Gamma=\emptyset .
$$

In fact, if $z \in A_{l_{1}} \ldots A_{l_{k-1}} q^{\prime}$ then, by (18), $z \in A_{l_{1}} \ldots A_{l_{k-1}} \stackrel{\circ}{ }$, and therefore $z \notin A_{h_{1}} \ldots A_{h_{k-1}} \stackrel{\circ}{ }$ for any $\left(h_{1}, \ldots, h_{k-1}\right) \neq\left(l_{1}, \ldots, l_{k-1}\right)$ - this is an easy consequence of (11). 
Step 4. Consider $y \equiv A_{l_{1}} \ldots A_{l_{k-1}} y^{\prime}$. Note that $q^{\prime}=y^{\prime}+q^{\prime \prime}$, where $q^{\prime \prime} \equiv-y^{\prime}+q^{\prime}$ is also an open cube. Define

$$
q^{\prime \prime \prime} \equiv\left\{r_{i}^{\left(l_{1}\right)} \ldots r_{i}^{\left(l_{k-1}\right)} \alpha \beta^{-1}\left(\eta_{j}^{\left(l_{1}\right)} \ldots \eta_{j}^{\left(l_{k-1}\right)} z_{j}\right)_{j=1}^{n}:\left(z_{j}\right)_{j=1}^{n} \in q^{\prime \prime}\right\},
$$

which is also an open cube, now with side-length $r_{i}^{\left(l_{1}\right)} \ldots r_{i}^{\left(l_{k-1}\right)} \alpha \beta^{-1}$ times that of $q^{\prime}$. The open cube $q \equiv y+q^{\prime \prime \prime}$ has the same side-length as $q^{\prime \prime \prime}$. We claim that

$$
y \in \bar{q}
$$

and

$$
q \subset A_{l_{1}} \ldots A_{l_{k-1}} q^{\prime} .
$$

Assertion (21) is clear, as $y^{\prime} \in \overline{q^{\prime}} \Rightarrow 0 \in \overline{-y^{\prime}+q^{\prime}} \Rightarrow 0 \in \overline{q^{\prime \prime \prime}} \Rightarrow y \in$ $\overline{y+q^{\prime \prime \prime}}$. For (22), observe that, given $z \in q, z=y+z^{\prime \prime \prime}$ for some $z^{\prime \prime \prime} \in q^{\prime \prime \prime}$, so $z=A_{l_{1}} \ldots A_{l_{k-1}} y^{\prime}+r_{i}^{\left(l_{1}\right)} \ldots r_{i}^{\left(l_{k-1}\right)} \alpha \beta^{-1}\left(\eta_{j}^{\left(l_{1}\right)} \ldots \eta_{j}^{\left(l_{k-1}\right)} z_{j}^{\prime \prime}\right)_{j=1}^{n}$ for some $\left(z_{j}^{\prime \prime}\right)_{j=1}^{n} \in q^{\prime \prime}$, that is,

$$
\begin{aligned}
z= & \left(\eta_{j}^{\left(l_{1}\right)} \ldots \eta_{j}^{\left(l_{k-1}\right)} r_{j}^{\left(l_{1}\right)} \ldots r_{j}^{\left(l_{k-1}\right)} y_{j}^{\prime}\right)_{j=1}^{n}+c_{l_{1}, \ldots, l_{k-1}} \\
& +\left(\eta_{j}^{\left(l_{1}\right)} \ldots \eta_{j}^{\left(l_{k-1}\right)} r_{j}^{\left(l_{1}\right)} \ldots r_{j}^{\left(l_{k-1}\right)} \lambda_{j} z_{j}^{\prime \prime}\right)_{j=1}^{n},
\end{aligned}
$$

where $\lambda_{j} \equiv r_{i}^{\left(l_{1}\right)} \ldots r_{i}^{\left(l_{k-1}\right)} \alpha \beta^{-1} /\left(r_{j}^{\left(l_{1}\right)} \ldots r_{j}^{\left(l_{k-1}\right)}\right)$ and $c_{l_{1}, \ldots, l_{k-1}}$ is the independent vector of the affine map $A_{l_{1}} \ldots A_{l_{k-1}}$ (as the $b_{l}$ in (4)). That is, any $z \in q$ can be written as

$$
\begin{aligned}
z & =\left(\eta_{j}^{\left(l_{1}\right)} \ldots \eta_{j}^{\left(l_{k-1}\right)} r_{j}^{\left(l_{1}\right)} \ldots r_{j}^{\left(l_{k-1}\right)}\left(y_{j}^{\prime}+\lambda_{j} z_{j}^{\prime \prime}\right)\right)_{j=1}^{n}+c_{l_{1}, \ldots, l_{k-1}} \\
& =A_{l_{1}} \ldots A_{l_{k-1}}\left(y^{\prime}+\left(\lambda_{j} z_{j}^{\prime \prime}\right)_{j=1}^{n}\right)
\end{aligned}
$$

for some $z^{\prime \prime} \equiv\left(z_{j}^{\prime \prime}\right)_{j=1}^{n} \in q^{\prime \prime}$. Since $0<\lambda_{j} \leq 1, j=1, \ldots, n$ (cf. (16) and the definitions of $\alpha$ and $\beta$, given at the beginning of Step 2), $z^{\prime \prime} \in q^{\prime \prime}$ and $0 \in \overline{q^{\prime \prime}}$, it follows that $\left(\lambda_{j} z_{j}^{\prime \prime}\right)_{j=1}^{n}$ is also in $q^{\prime \prime}$ (recall that we are only considering cubes with sides parallel to the axes) and (22) now follows easily.

As a consequence of (20) and (22), we have

$$
q \cap \Gamma=\emptyset \text {. }
$$

Step 5. Observe now that, given any $z \in \bar{q}$,

$$
\begin{aligned}
|z-x| & \leq|z-y|+|y-x| \\
& \leq \sqrt{n} r_{i}^{\left(l_{1}\right)} \ldots r_{i}^{\left(l_{k-1}\right)} \alpha \beta^{-1}+r_{i}^{\left(l_{1}\right)} \ldots r_{i}^{\left(l_{k-1}\right)}\left|y_{i}^{\prime}-x_{i}^{\prime}\right| \\
& \leq r_{i}^{\left(l_{1}\right)} \ldots r_{i}^{\left(l_{k-1}\right)}\left(\sqrt{n} \alpha \beta^{-1}+1\right) \\
& \leq r_{i}^{\left(l_{1}\right)} \ldots r_{i}^{\left(l_{k-1}\right)} r_{i}^{\left(l_{k}\right)} \alpha^{-1}\left(\sqrt{n} \alpha \beta^{-1}+1\right) \\
& \leq \theta r \alpha^{-1}\left(\sqrt{n} \alpha \beta^{-1}+1\right)=r
\end{aligned}
$$


(we have used (15) in the last inequality), so that

$$
\bar{q} \subset B(x, r) .
$$

Finally, let $c$ stand for the centre of $q$. Then the ball $B(c, \ell(q) / 2)$ is in $\bar{q}$, therefore also in $B(x, r)$, and has radius $r_{i}^{\left(l_{1}\right)} \ldots r_{i}^{\left(l_{k-1}\right)} \alpha \beta^{-1} \ell\left(q^{\prime}\right) / 2$, which, by (16) and the first phrase in Step 1, is strictly bounded below by $\theta r \alpha \beta^{-1} \mu / 2$. Hence

$$
B\left(c, \theta \alpha \beta^{-1} \mu r / 2\right) \subset q \subset B(x, r)
$$

and, due to (23),

$$
B\left(c, \theta \alpha \beta^{-1} \mu r / 2\right) \cap \Gamma=\emptyset .
$$

This accomplishes the proof that $\Gamma$ satisfies the ball condition, with $\eta=\theta \alpha \beta^{-1} \mu / 2$ in Definition 3.1.

COROLlary 5.7. Let $\Gamma$ be an anisotropic self-affine fractal. Let $A_{l}, l=$ $1, \ldots, N$, denote the affine contractions (13) which generate $\Gamma$. Assume that there is $k_{0} \in \mathbb{N}$ such that for every $i \in\{1, \ldots, n\}$, every $l_{1}, \ldots, l_{k_{0}} \in$ $\{1, \ldots, N\}$ and every $x \equiv\left(x_{j}\right)_{j=1}^{n} \in A_{l_{1}} \ldots A_{l_{k_{0}}} Q$, there exist $y \equiv\left(x_{1}, \ldots\right.$, $\left.x_{i-1}, y_{i}, x_{i+1}, \ldots, x_{n}\right)$ and an open cube $q \subset Q$ such that $y \in \bar{q}$ and

$$
q \cap A^{k_{0}} Q=\emptyset \text {. }
$$

Then $\Gamma$ is NST.

Proof. Let $\Gamma^{\prime}$ be the self-affine fractal generated by the family $\left\{A_{l_{1} \ldots l_{k_{0}}}\right.$ : $\left.l_{1}, \ldots, l_{k_{0}} \in\{1, \ldots, N\}\right\}$ of affine contractions $A_{l_{1} \ldots l_{k_{0}}} \equiv A_{l_{1}} \ldots A_{l_{k_{0}}}$. It is not difficult to see that $\Gamma^{\prime}$ satisfies the conditions of Theorem 5.6, so it is NST. Now just note that $\Gamma=\Gamma^{\prime}$.

In the following examples of anisotropic self-affine fractals in $\mathbb{R}^{2}$, the affine contractions $A_{l}$ are represented by means of the images $A_{l} Q$, which, in turn, are represented by the shaded rectangles in the corresponding figure.

EXAMPLE 1. The anisotropic fractal generated by the affine contractions represented in Figure 1 is NST.

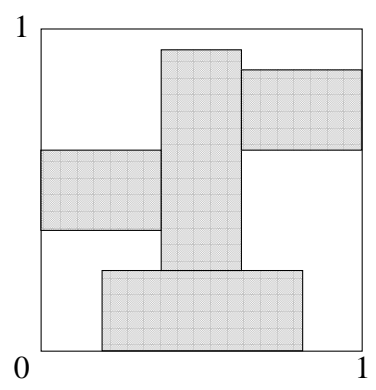

Fig. 1 
EXAMPle 2. An anisotropic fractal to which the corollary (with $k_{0}$ $=3$ ) - but not the theorem - can be applied is generated by the affine contractions represented in Figure 2.

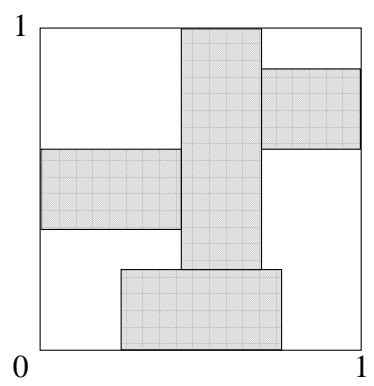

Fig. 2

We can weaken the hypotheses of Theorem 5.6 if we deal with a special subclass of anisotropic fractals. We start with some preparations:

With the same notations as in (13), given $A_{l}, l=1, \ldots, N$, if $i \in$ $\{1, \ldots, n\}$ is such that $r_{i}^{(l)}=\min _{1 \leq j \leq n} r_{j}^{(l)}$, we say that the $i$-axis is an axis of strongest contraction (for the affine contraction $A_{l}$ ).

Definition 5.8. An anisotropic fractal is called a CASC-fractal if the affine contractions of a generating family $\left\{A_{l}: l \in\{1, \ldots, N\}\right\}$ have a Common Axis of Strongest Contraction, that is, if there is $i \in\{1, \ldots, n\}$ such that for all $l=1, \ldots, N, \min _{1 \leq j \leq n} r_{j}^{(l)}=r_{i}^{(l)}$.

TheOREm 5.9. Let $\Gamma$ be a $C A S C$-fractal. Let $A_{l}, l=1, \ldots, N$, denote the affine contractions (13) which generate $\Gamma$ and let the $i$-axis be a common axis of strongest contraction. Assume that, for every $l \in\{1, \ldots, N\}$ and every $x \equiv\left(x_{j}\right)_{j=1}^{n} \in A_{l} Q$, there exist $y \equiv\left(x_{1}, \ldots, x_{i-1}, y_{i}, x_{i+1}, \ldots, x_{n}\right)$ and an open cube $q \subset Q$ such that $y \in \bar{q}$ and

$$
q \cap \bigcup_{\ell=1}^{N} A_{\ell} Q=\emptyset .
$$

Then $\Gamma$ is NST.

Proof. Since the proof is very similar to the proof of Theorem 5.6, we only point out the necessary modifications.

Step 1 is the same.

Step 2: To begin with, $\theta$ is now defined as $\alpha /(\sqrt{n}+1)$. The parameter $\alpha$ can be defined more simply as $\min \left\{r_{i}^{(l)}: l \in\{1, \ldots, N\}\right\}$, because of the role played by the number $i$, which is here given from the very beginning. As to $\beta$, it is enough now to define it by $\max \left\{r_{i}^{(l)}: l \in\{1, \ldots, N\}\right\}$. Since $i$ is given a priori, in this step we can fix $j=i$, so that there is no point in considering 
$k_{0}$ - or, if one prefers, just take $k_{0}=k_{i}$-and one should suppress the part where the $i$ is chosen. We again use the letter $k$ to denote $k_{0}$ (that is, $k_{i}$ ), and the last part of Step 2 should now read: in what follows the $k \in \mathbb{N}$ is chosen such that

$$
\begin{gathered}
r_{i}^{\left(l_{1}\right)} \ldots r_{i}^{\left(l_{k}\right)} \leq \theta r \\
r_{i}^{\left(l_{1}\right)} \ldots r_{i}^{\left(l_{k-1}\right)}>\theta r .
\end{gathered}
$$

Step 3 is the same.

Step 4: Suppress the factor $\alpha \beta^{-1}$ in all considerations. In particular, $\lambda_{j}$ now reads $r_{i}^{\left(l_{1}\right)} \ldots r_{i}^{\left(l_{k-1}\right)} /\left(r_{j}^{\left(l_{1}\right)} \ldots r_{j}^{\left(l_{k-1}\right)}\right)$. The justification that $0<\lambda_{j} \leq 1$ must be accordingly modified (but it is trivial).

Step 5: Suppress the factor $\alpha \beta^{-1}$ in all considerations.

Corollary 5.10. Let $\Gamma$ be a CASC-fractal. Let $A_{l}, l=1, \ldots, N$, denote the affine contractions (13) which generate $\Gamma$ and let the $i$-axis be a common axis of strongest contraction. Assume that there is $k_{0} \in \mathbb{N}$ such that for every $l_{1}, \ldots, l_{k_{0}} \in\{1, \ldots, N\}$ and every $x \equiv\left(x_{j}\right)_{j=1}^{n} \in A_{l_{1}} \ldots A_{l_{k_{0}}} Q$, there exist $y \equiv\left(x_{1}, \ldots, x_{i-1}, y_{i}, x_{i+1}, \ldots, x_{n}\right)$ and an open cube $q \subset Q$ such that $y \in \bar{q}$ and

$$
q \cap A^{k_{0}} Q=\emptyset .
$$

Then $\Gamma$ is NST.

This follows just as Corollary 5.7, with Theorem 5.9 replacing Theorem 5.6.

EXAMPle 3. A CASC-fractal to which Theorem 5.9-but not Theorem 5.6 nor Corollary 5.7 - can be applied is generated by the affine contractions represented in Figure 3.

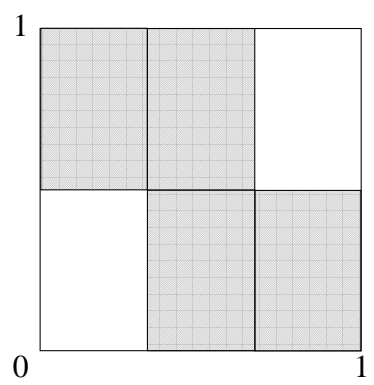

Fig. 3

EXAmple 4. A CASC-fractal to which Corollary 5.10-but not Theorems 5.6 and 5.9 nor Corollary 5.7 - can be applied is generated by the affine contractions represented in Figure 4. 


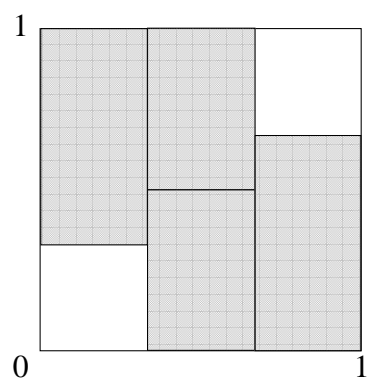

Fig. 4

Of course, Theorems 5.6, 5.9 and Corollaries 5.7, 5.10 only give sufficient conditions for an anisotropic fractal to be NST:

EXAMPLE 5. The 2-dimensional Cantor set generated by the similarities represented in Figure 5 is a CASC-fractal to which none of those results apply; nevertheless, it is NST (cf. Proposition 5.3 and comments afterwards).

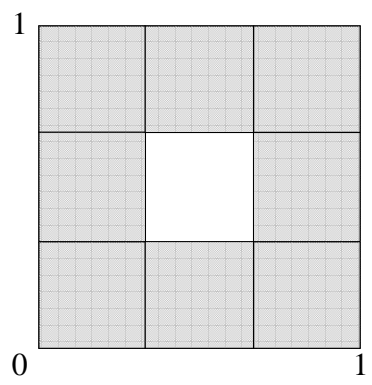

Fig. 5

Some conditions must, however, be imposed in order for an anisotropic (even if CASC-) fractal to be NST, as the following example shows.

Example 6. Consider the anisotropic 2-dimensional Cantor-like set $\Gamma$ generated by the affine contractions represented in Figure 6.

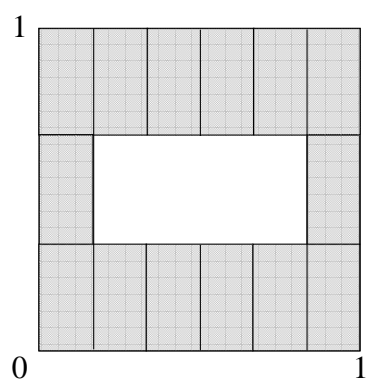

Fig. 6 
Clearly, this is even a CASC-fractal. We shall see, however, that it is not NST:

First observe that, denoting by $R$ the free space open rectangle in the

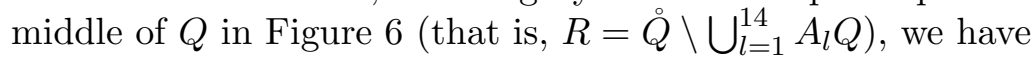

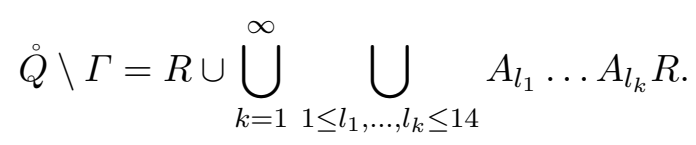

Calling $A_{l_{1}} \ldots A_{l_{k}} R$ free space rectangles of the $k$ th generation and using the usual convention to denote coordinates in dimension 2 , note that, below the line $y=3^{-(k+1)}$ in Figure 6 there can be no free space rectangles of generation $k$ popping in. On the other hand, the basis of each free space rectangle of generation $k$ has length $(2 / 3) 6^{-k}$, so that the higher the generation, the smaller the length of the bases of its free space rectangles.

Consider then the sequence $\left(\left(1 / 3,3^{-(k+1)}\right)\right)_{k \in \mathbb{N}}$ of points of $\Gamma$ and the sequence of closed balls $B_{k}$ of radius $3^{-(k+1)}$ centred at those points. Observe that each such ball is contained in $Q$ and stays below the line $y=3^{-k}$. Therefore, the only points of that ball which are not in $\Gamma$ must be in free space rectangles of $k$ th or higher generation. Since these are pairwise disjoint, it is not possible to find a closed ball contained in $B_{k} \backslash \Gamma$ and with radius greater than $(1 / 3) 6^{-k}$. And since the ratio $(1 / 3) 6^{-k} / 3^{-(k+1)}$, that is, $2^{-k}$, tends to 0 as $k$ goes to infinity, it is not possible to find $\left.\eta \in\right] 0,1[$ such that, for any $k \in \mathbb{N}$ and for some $y_{k} \in \mathbb{R}^{2}, B\left(y_{k}, \eta 3^{-(k+1)}\right) \subset B_{k}$ and $B\left(y_{k}, \eta 3^{-(k+1)}\right) \cap \bar{\Gamma}=\emptyset$.

This shows that such a $\Gamma$ does not satisfy the ball condition. That $\Gamma$ is not NST follows now from Remark 5.5.

REMARK 5.11. (i) This also serves as an example of a fractal satisfying the open set condition (see (9)) but which is not in NST, so that in this way we complement what has been said after Remark 3.5.

(ii) The $\Gamma$ of Example 6 is not a $d$-set. This is clear for $d<n$, due to Proposition 4.3; for $d=n$ it is also clear from the fact that $|\Gamma|=0$ (Lemma 5.4).

(iii) Consider the open set $\Gamma^{\mathrm{c}}$, with $\Gamma$ the fractal in Example 6. From Proposition 3.2 it follows that $\Gamma^{\mathrm{c}} \notin \mathrm{NST}$. Since $|\Gamma|=0, \Gamma^{\mathrm{c}}$ is an open $n$-set and therefore we have found another example (less trivial than the one exhibited after Remark 4.4) of an open $n$-set which is not NST.

EXAMPLE 7. From Theorem 5.9 it is clear that the modification of Figure 6 given in Figure 7 yields, in the usual way, a representation of a CASCfractal which is NST. 


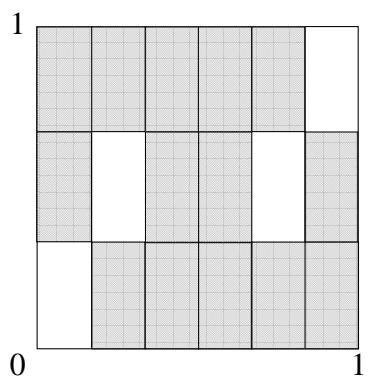

Fig. 7

REMARK 5.12. This together with Example 6 shows that, for anisotropic fractals, the property of being NST or not has nothing to do with the socalled affine dimension of fractals, as defined in [9]. The fractals in Examples 6 and 7 have the same affine dimension (just because the number of shaded rectangles of the same size is the same, irrespective of their positions) namely $\left(2+2 \log _{2} 7\right) /\left(1+2 \log _{2} 3\right)$ - while one is NST and the other is not (the results proved above show that the relative position of the shaded rectangles can make a difference).

Acknowledgements. We would like to thank the referee for drawing our attention to the notion of porosity and for the suggestions for the improvement of the text.

\section{References}

[1] K. J. Falconer, The Geometry of Fractal Sets, Cambridge Univ. Press, Cambridge, 1985.

[2] - Fractal Geometry, Wiley, Chichester, 1990.

[3] M. Frazier and B. Jawerth, A discrete transform and decompositions of distribution spaces, J. Funct. Anal. 93 (1990), 34-170.

[4] A. Jonsson, Atomic decomposition of Besov spaces on closed sets, in: Function Spaces, Differential Operators and Nonlinear Analysis, H.-J. Schmeisser and H. Triebel (eds.), Teubner, Stuttgart, 1993, 285-289.

[5] A. Jonsson and H. Wallin, Function Spaces on Subsets of $\mathbb{R}^{n}$, Math. Reports 2, Harwood, London, 1984.

[6] P. Mattila, Geometry of Sets and Measures in Euclidean Spaces, Cambridge Univ. Press, Cambridge, 1995.

[7] V. G. Maz'ja, Sobolev Spaces, Springer, Berlin, 1985.

[8] - Classes of Domains, Measures and Capacities in the Theory of Differentiable Functions, Encyclopaedia Math. Sci. 26, Springer, Berlin, 1991.

[9] H. Triebel, Fractals and Spectra, Birkhäuser, Basel, 1997.

[10] H. Triebel and H. Winkelvoss, A Fourier analytical characterization of the Hausdorff dimension of a closed set and related Lebesgue spaces, Studia Math. 121 (1996), 149-166. 
[11] H. Triebel and H. Winkelvoss, Intrinsic atomic characterizations of function spaces on domains, Math. Z. 221 (1996), 647-673.

Departamento de Matemática

Universidade de Aveiro

3810-193 Aveiro, Portugal

E-mail: acaetano@mat.ua.pt

Received 18 September 2000; in revised form 12 February 2001 